\title{
Applications of High Precision STEM Imaging to Structurally Complex Materials
}

\author{
Jie Feng, ${ }^{1}$ Chenyu Zhang, ${ }^{1}$ Dan Zhou, ${ }^{1}$ Zhongnan Xu, ${ }^{1}$ Dane Morgan, ${ }^{1}$ and Paul M. Voyles ${ }^{1}$ \\ 1. Department of Materials Science and Engineering, University of Wisconsin-Madison, Madison, WI \\ USA
}

\begin{abstract}
Aberration corrected electron microscopy has made Ångstrom resolution imaging routine in labs across the world. One of the next frontiers is improving the quantitative reliability of data about materials we can extract from aberration-corrected images. We have developed a methodology based on non-rigid registration of a series of short exposure time STEM images which we call high precision STEM [1]. High precision STEM results in images in which random noise is suppressed well below the scattering generated by a single atom, and the position of atomic columns in the image can be determine to within $<1 \mathrm{pm}$ [1]. Other related approaches yield similar results [2, 3]. This paper presents two applications of high precision STEM imaging to the structurally complex materials with a varying number of degrees of freedom in the atomic structure.
\end{abstract}

The first example is high precision STEM imaging of single La vacancies $\left(\mathrm{V}_{\mathrm{La}}\right)$ in $\mathrm{LaMnO}_{3}$. We treat vacancies as defects with known atomic structure, derived from density functional theory calculations. As a result, the unknown parameters are the atomic coordinates of the missing La atom. Using high precision STEM, we can image $\mathrm{V}_{\mathrm{La}}$ both from the reduction in the intensity of the atomic column containing the vacancy and from the displacement of the neighboring La-containing columns in toward the vacancy. For relatively thin TEM sample, $\sim 10 \mathrm{~nm}$ thick, the visibility of a single $\mathrm{V}_{\mathrm{La}}$ is well above the $\sim 1 \%$ intensity uncertainty in high precision STEM for any depth of the vacancy. In three dimensions, the neighbor La displacements are $20-30 \mathrm{pm}$, but only a maximum of 8 pm survives the two dimensional projection and probe channeling of the STEM imaging process, and when the $\mathrm{V}_{\mathrm{La}}$ is near the exit surface, the displacements in the image are nearly zero. Both the visibility and the displacements depend on the depth of the $\mathrm{V}_{\mathrm{La}}$ through channeling of the electron probe, but the depth dependencies are not the same. Therefore, by combining information about the visibility and displacements, we can determine the depth of the vacancy, in addition the two dimensional position available directly from the image.

Figure 1 shows an example high precision STEM image of $\mathrm{LaMnO}_{3}$, with the probability of selected column containing a vacancy superimposed. The probability is evaluated by comparing the experimental visibility and displacements to a library of simulations using a Bayesian model. The library of simulations give the probability of observing a particular visibility and set of displacements, given a vacancy at a particular depth and an estimate of experimental errors. Bayes' theorem lets us find the inverse probability, the probability that a vacancy exists at a particular depth, given a set of observations.

The second example is high precision STEM imaging combined with computational structural refinement to solve the structure of nanoparticles. Nanoparticles have many more degrees of freedom, due to the need to specify the shape of the particle and the position of atoms on the surface which generally do not not occupy exact lattice positions. High precision STEM images provide reliable data on the number of atoms in each atomic column and the position of surface columns, but deriving the full three dimensional structure from a single projection image is typically not possible. Our approach is to combine information from experimental images with a calculation of the total particle energy from an 
empirical interatomic potential into a cost function $C(s)=\chi^{2}\left(I_{s}, I_{e}\right)+\alpha E(s)$, where $s$ is a structure, $\chi^{2}$ is the squared difference of the simulated image $I_{s}$ and the experimental image $I_{e}$, computed pixel-by-pixel, $E$ is the energy of a structure, and $\alpha$ is a scaling parameter. We then minimize $C(s)$ using a genetic algorithm [4].

Figure 2 shows an experimental image of a Au nanoparticle, the derived structure of the particle, and the simulated image from the derived structure. The particle is pancake-shaped, not spherical as might be expected from the 2D image. The derived structure also finds atoms on the particle surface that are not well-localized in the original data, potentially due to beam damage. This structure refinement method has been recently generalized to treat alloy particles and tested on Pt-Mo nanocatalysts [5].

\section{References:}

[1] A. B. Yankovich, et al., Nat. Commun. 5, (2014), 4155

[2] X. Sang and J. M. Lebeau, Ultramicroscopy 138, (2014), 28

[3] C. Ophus, J. Ciston, and C. T. Nelson, Ultramicroscopy 162, (2016), 1.

[4] M. Yu et al., ACS Nano 10, (2016), 4031

[5] High precision STEM method development and imaging of vacancies was supported by the U.S. Department of Energy (DE-FG02-08ER46547). Reverse structure determination was supported by the U.S. National Science Foundation (DMR-1332851).
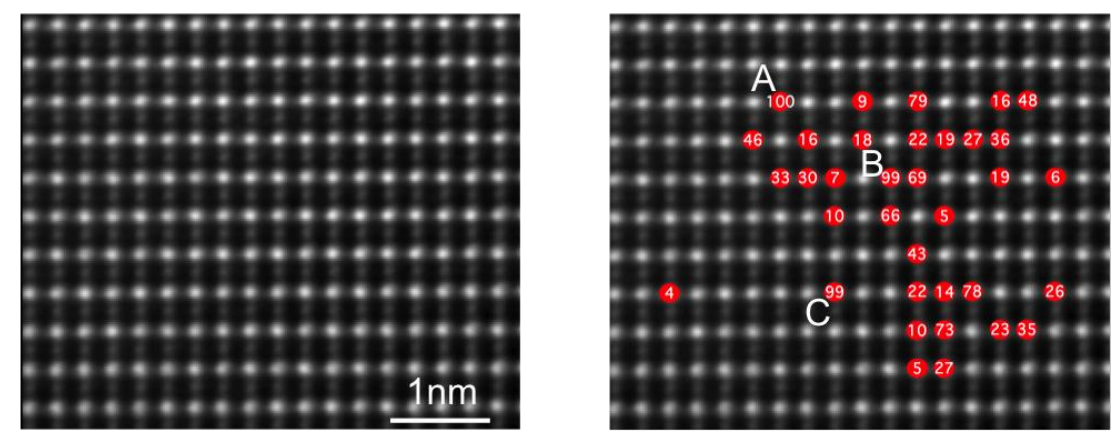

Figure 1. (left) Example high precision STEM image of $\mathrm{LaMnO}_{3}$. (right) Percentage chance of the labeled columns containing a vacancy, according to the Bayesian model.

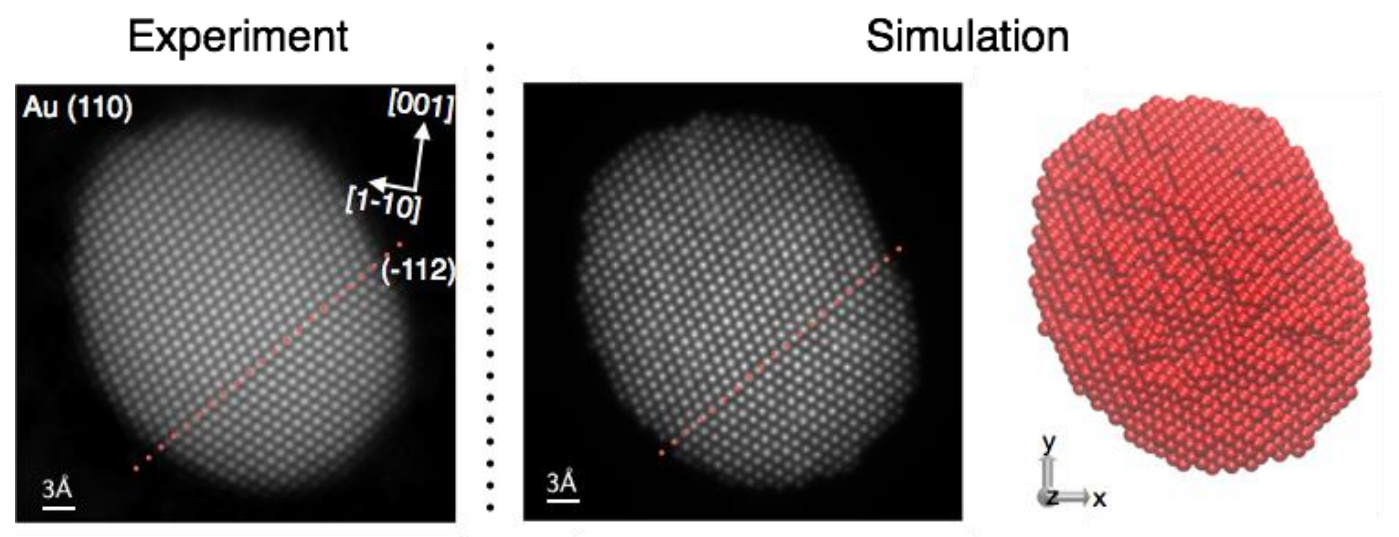

Figure 2. (left) Experimental high-precision STEM image of a Au nanoparticle. (middle) the simulated image of the derived structure. (right) the derived structure. 